UCRHEP-T115

$\mathrm{MAD} / \mathrm{PH} / 792$

September 1993

\title{
New Supersymmetric Electroweak Gauge Model with Natural Baryon-Number Conservation
}

\author{
Ernest Ma \\ Department of Physics, University of California, Riverside, California 92521 \\ and \\ Department of Physics, University of Wisconsin, Madison, Wisconsin 53706
}

\begin{abstract}
Baryon number (B) is automatically conserved in the standard $\mathrm{SU}(2) \times \mathrm{U}(1)$ electroweak gauge model, but not in its supersymmetric extension where it must be imposed as an extra condition. This undesirable feature is avoided in the conventional left-right supersymmetric extension, but then flavor-changing neutral currents (FCNC) are not naturally suppressed. A new version is proposed with automatic B conservation as well as FCNC suppression (except for $u$ quarks through scalar exchange.)
\end{abstract}


In the standard $\mathrm{SU}(2) \times \mathrm{U}(1)$ electroweak gauge model, because of its assumed particle content and the requirement of gauge invariance, it is not possible to write down renormalizable interaction terms which violate the conservation of baryon number (B). This is a very desirable feature of the model since B conservation is experimentally known to be very well respected. On the other hand, if the model is extended to include supersymmetry, then such terms are in general allowed. Consider the quark and lepton superfields. In a notation where only the left chiral projections are counted, they transform under $\mathrm{SU}(3) \times \mathrm{SU}(2) \times \mathrm{U}(1)$ as follows: $Q \equiv(u, d) \sim(3,2,1 / 6), u^{c} \sim(\overline{3}, 1,-2 / 3), d^{c} \sim(\overline{3}, 1,1 / 3), L \equiv(\nu, e) \sim(1,2,-1 / 2)$, and $e^{c} \sim(1,1,1)$, where the family index has been suppressed. In addition, there must be two Higgs superfields $\Phi_{1,2}$ transforming as $(1,2, \mp 1 / 2)$ respectively. The desirable allowed terms in the superpotential are then $\Phi_{1} Q d^{c}, \Phi_{2} Q u^{c}$, and $\Phi_{1} L e^{c}$, which supply the quarks and leptons with masses as the neutral scalar components of $\Phi_{1,2}$ acquire nonzero vacuum expectation values $v_{1,2}$. However, the terms $u^{c} d^{c} d^{c}, L Q d^{c}$, and $L L e^{c}$ are also allowed a priori and they violate the conservation of B as well as that of lepton number (L). Note also that $\Phi_{1}$ and $L$ are indistinguishable by their transformations alone.

To obtain a realistic model, the usual solution is to impose B and L conservation as an extra condition. For example, if a discrete $\mathrm{Z}_{2}$ symmetry is assumed such that $\Phi_{1,2}$ are even, but all other superfields are odd, then only the desirable terms are retained. Since both $\mathrm{B}$ and $\mathrm{L}$ are now conserved by assumption, a quantity $\mathrm{R} \equiv(-1)^{2 \mathrm{j}+3 \mathrm{~B}+\mathrm{L}}$ can be used to distinguish particles $(\mathrm{R}=+1)$ from superparticles $(\mathrm{R}=-1)$. This has become such common practice that its origin as an imposed condition is often accepted without much attention. However, as long as there is a need to impose some extra symmetry on the supersymmetric standard model, the choice of that symmetry becomes somewhat arbitrary. For example, if $L$ and $e^{c}$ are even instead of odd in the above, then the $u^{c} d^{c} d^{c}$ terms are still forbidden, but the $L Q d^{c}$ and $L L e^{c}$ terms are allowed. Hence $\mathrm{B}$ is conserved, but $\mathrm{L}$ is not. Such models 
have been studied in the past [1] and limits on the various possible Yukawa couplings have been obtained, usually under certain restrictive assumptions. It is also possible to refine the imposed condition so that instead of preserving all three lepton numbers, i.e. $\mathrm{L}^{e}$, $\mathrm{L}^{\mu}$, and $\mathrm{L}^{\tau}$, only one linear combination (say $\mathrm{L}^{e}-\mathrm{L}^{\mu}$ ) or two (say $\mathrm{L}^{e}$ and $\mathrm{L}^{\mu}$ ) are conserved.[2] Finally, it may be assumed that $Q, u^{c}$, and $d^{c}$ are even instead of odd, then $\mathrm{L}$ is conserved but $\mathrm{B}$ is not. This may also lead to some rather interesting phenomenology. [3]

Given that the terms $u^{c} d^{c} d^{c}, L Q d^{c}$, and $L L e^{c}$ are problematic in the supersymmetric standard model, it is natural to ask whether such terms can be avoided automatically in a larger theory. The answer is yes if we simply take the conventional left-right supersymmetric extension of the standard model. The gauge group is now $\mathrm{SU}(3) \times \mathrm{SU}(2) \times \mathrm{SU}(2) \times \mathrm{U}(1)$. The quarks and leptons are

$$
\begin{array}{cl}
Q \equiv(u, d) \sim(3,2,1,1 / 6), & Q^{c} \equiv\left(d^{c}, u^{c}\right) \sim(\overline{3}, 1,2,-1 / 6), \\
L \equiv(\nu, e) \sim(1,2,1,-1 / 2), & L^{c} \equiv\left(e^{c}, N^{c}\right) \sim(1,1,2,1 / 2) .
\end{array}
$$

Hence terms involving three such superfields are not possible in the superpotential because of gauge invariance, and the automatic conservation of $\mathrm{B}$ and $\mathrm{L}$ is achieved. On the other hand, realistic quark and lepton masses require the existence of two bidoublet Higgs superfields $\Phi_{1,2} \sim(1,2,2,0)$ with four nonzero vacuum expectation values, and flavor-changing neutral currents (FCNC) are not naturally suppressed. It is perhaps for this reason that no detailed analysis of the conventional left-right supersymmetric model exists in the literature.

An alternative left-right supersymmetric model with natural flavor conservation can be constructed [4] if we add an extra quark $h$ of charge $-1 / 3$ so that we may repalce $d^{c}$ by $h^{c}$ in $Q^{c}$, leaving $h$ and $d^{c}$ as singlets. In that case, each scalar vacuum expectation value is matched to a different quark type, and the natural suppression of FCNC is assured. However, an extra discrete symmetry is still required to distinguish $h$ from $d$ and it is this same symmetry which forbids the undesirable terms $Q Q h$ and $Q^{c} Q^{c} d^{c}$. 
Consider instead now the addition of an extra quark $x$ of charge $2 / 3$ with no extra symmetry. Let

$$
x \sim(3,1,1,2 / 3), \quad x^{c} \sim(\overline{3}, 1,1,-2 / 3),
$$

then it is again not possible to have terms in the superpotential involving three quark or lepton superfields, and the desirable feature of automatic B and L conservation is achieved. The Higgs superfields are

$$
\Phi_{12} \equiv\left(\begin{array}{cc}
\overline{\phi_{1}^{0}} & \phi_{2}^{+} \\
-\phi_{1}^{-} & \phi_{2}^{0}
\end{array}\right) \sim(1,2,2,0)
$$

and

$$
\Phi_{3} \equiv\left(\overline{\phi_{3}^{0}},-\phi_{3}^{-}\right) \sim(1,1,2,-1 / 2), \quad \Phi_{4} \equiv\left(\phi_{4}^{+}, \phi_{4}^{0}\right) \sim(1,1,2,1 / 2)
$$

Note that both $\Phi_{3}$ and $\Phi_{4}$ are singelts under the standard $\mathrm{SU}(2) \times \mathrm{U}(1)$ and the above structure is anomaly-free. The allowed Yukawa terms are then

$$
\Phi_{12} Q Q^{c}=\overline{\phi_{1}^{0}} d d^{c}+\phi_{1}^{-} u d^{c}+\phi_{2}^{0} u u^{c}-\phi_{2}^{+} d u^{c}
$$

and

$$
\Phi_{3} x Q^{c}=\overline{\phi_{3}^{0}} x u^{c}+\phi_{3}^{-} x d^{c}
$$

Since $x$ and $x^{c}$ are singlets, there is also an allowed gauge-invariant mass term $x x^{c}$. Let $\left\langle\phi_{1,2,3}^{0}\right\rangle=v_{1,2,3}$, then the $6 \times 6$ mass matrix linking $(u, x)$ with $\left(u^{c}, x^{c}\right)$ is given by

$$
\mathcal{M}_{u x}=\left[\begin{array}{cc}
v_{2} v_{1}^{-1} \mathcal{M}_{d} & 0 \\
\mathcal{M}_{3} & \mathcal{M}_{x}
\end{array}\right]
$$

where the $3 \times 3$ matrices $\mathcal{M}_{d}$ and $\mathcal{M}_{x}$ can be defined to be diagonal, and $\mathcal{M}_{3}$ is proportional to $v_{3}$. Note that $\Phi_{4}$ is not involved at all and it will be assumed here that $\left\langle\phi_{4}^{0}\right\rangle=0$. The mixing of $u$ and $x$ is determined by the matrix

$$
\mathcal{M}_{u x} \mathcal{M}_{u x}^{\dagger}=\left[\begin{array}{cc}
v_{2}^{2} v_{1}^{-2} \mathcal{M}_{d} \mathcal{M}_{d}^{\dagger} & v_{2} v_{1}^{-1} \mathcal{M}_{d} \mathcal{M}_{3}^{\dagger} \\
v_{2} v_{1}^{-1} \mathcal{M}_{3} \mathcal{M}_{d}^{\dagger} & \mathcal{M}_{3} \mathcal{M}_{3}^{\dagger}+\mathcal{M}_{x} \mathcal{M}_{x}^{\dagger}
\end{array}\right]
$$


and since $\mathcal{M}_{d}$ should be very much smaller than $\mathcal{M}_{3}$ and $\mathcal{M}_{x}$, it is clear that $u-x$ mixing is very small and can be safely neglected. On the other hand, the mass matrix for the $u$ quarks is given by

$$
\mathcal{M}_{u} \mathcal{M}_{u}^{\dagger}=v_{2}^{2} v_{1}^{-2} \mathcal{M}_{d}\left[1-\mathcal{M}_{3}^{\dagger}\left(\mathcal{M}_{3} \mathcal{M}_{3}^{\dagger}+\mathcal{M}_{x} \mathcal{M}_{x}^{\dagger}\right)^{-1} \mathcal{M}_{3}\right] \mathcal{M}_{d}^{\dagger}
$$

which is in general nondiagonal and can easily be phenomenologically correct even if $u-x$ mixing is very small. The mixing of $u^{c}$ and $x^{c}$ is determined by the matrix $\mathcal{M}_{u x}^{\dagger} \mathcal{M}_{u x}$ and can be quite large because $\mathcal{M}_{3}$ and $\mathcal{M}_{x}$ should be comparable in magnitude for a realistic $\mathcal{M}_{u}$ to be obtained. However, because $u^{c}$ and $x^{c}$ transform identically under the standard $\mathrm{SU}(2) \times \mathrm{U}(1)$, this mixing is observable at the electroweak energy scale only through scalar interactions. Nevertheless, this means that FCNC for the $u$ quarks exist and they should be searched for in processes such as $D^{0}-\overline{D^{0}}$ mixing.

In the leptonic sector, the analogous Yukawa term to Eq. (6) is

$$
\Phi_{12} L L^{c}=\overline{\phi_{1}^{0}} e e^{c}+\phi_{1}^{-} \nu e^{c}+\phi_{2}^{0} \nu N^{c}-\phi_{2}^{+} e N^{c}
$$

which by itself would imply that $\nu$ and $N^{c}$ should form a Dirac neutrino with mass equal to $v_{2} v_{1}^{-1} m_{e}$. That is of course unrealistic. The usual solution of this problem in the conventional left-right model is to introduce a complex Higgs triplet which provides a large Majorana mass for $N^{c}$, thus breaking $\mathrm{L}$ in the process. Here it is simpler just to add a singlet superfield $N$ (with zero vacuum expectation value) and the Yukawa term analogous to Eq. (7) is

$$
\Phi_{3} L^{c} N=\overline{\phi_{3}^{0}} N^{c} N+\phi_{3}^{-} e^{c} N
$$

Noting that a gauge-invariant Majorana mass for $N$ is allowed, the $9 \times 9$ Majorana mass matrix spanning $\nu, N^{c}$, and $N$ is then given by

$$
\mathcal{M}=\left[\begin{array}{ccc}
0 & v_{2} v_{1}^{-1} \mathcal{M}_{\ell} & 0 \\
v_{2} v_{1}^{-1} \mathcal{M}_{\ell} & 0 & \mathcal{M}_{3}^{\prime} \\
0 & \mathcal{M}_{3}^{\prime} & \mathcal{M}_{N}
\end{array}\right]
$$


where the $3 \times 3$ mass matrices $\mathcal{M}_{\ell}$ and $\mathcal{M}_{N}$ can be defined to be diagonal, and $\mathcal{M}_{3}^{\prime}$ is proportional to $v_{3}$. Under the reasonable assumption $v_{2} v_{1}^{-1} \mathcal{M}_{\ell} \ll \mathcal{M}_{3}^{\prime} \ll \mathcal{M}_{N}$, the neutrino mass matrix is then of the double-see-saw form:

$$
\mathcal{M}_{\nu}=v_{2}^{2} v_{1}^{-2} \mathcal{M}_{\ell} \mathcal{M}_{3}^{\prime-1} \mathcal{M}_{N} \mathcal{M}_{3}^{\prime-1} \mathcal{M}_{\ell}
$$

Naturally small neutrino masses as well as mixing among the three neutrinos are now possible and a realistic lepton sector is obtained.

Recall that in the supersymmetric standard model, one of the Higgs superfields, namely $\Phi_{1}$, is identical to the doublet lepton superfields $L$ in its transformations. Now there is no confusion with $L$, but instead we have $\Phi_{4}$ and $L^{c}$ which transform in the same way. This means that the terms $\Phi_{3} \Phi_{4}, \Phi_{3} L^{c}, \Phi_{3} \Phi_{4} N$, and $\Phi_{12} \Phi_{4} L$ are allowed. However, we can always redefine $\Phi_{4}$ and $L^{c}$ so that the $\Phi_{3} L^{c}$ term is absent. Now since $\left\langle\phi_{3}^{0}\right\rangle=v_{3}$ but neither $\Phi_{4}$ nor $L^{c}$ has a vacuum expectation value, the $5 \times 5$ mass matrix linking $\left(\ell, \psi_{3}^{-}, \tilde{w}_{R}^{-}\right)$with $\left(\ell^{c}, \psi_{4}^{+}, \tilde{w}_{R}^{+}\right)$, where $\tilde{w}_{R}^{ \pm}$are the charged $\mathrm{SU}(2)_{\mathrm{R}}$ gauginos and $\psi_{3,4}^{\mp}$ are the corresponding higgsinos, is of the form

$$
\mathcal{M}=\left[\begin{array}{ccc}
\mathcal{M}_{\ell} & \mathcal{M}_{12} & 0 \\
0 & \mu_{34} & M_{W_{R}} \sqrt{2} \\
0 & 0 & M_{R}
\end{array}\right]
$$

where $\mathcal{M}_{12}$ is a $3 \times 1$ matrix coming from the $\Phi_{12} \Phi_{4} L$ term, and $M_{R}$ is an allowed $\mathrm{SU}(2)_{\mathrm{R}}$ gauge-invariant term which softly breaks the supersymmetry. Since $\mu_{34}$ is expected to be very much greater than $\mathcal{M}_{12}$ in magnitude, the mixing of $\ell$ with $\psi_{3}^{-}$and $\tilde{w}_{R}^{-}$can easily be made negligible and lepton universality at the electroweak energy scale is maintained. However, the diagonalization of the above mass matrix will involve some small mixing of the leptons with the charginos so that flavor-changing neutral currents will be present, but only at a very much reduced level.

Because of the choice of gauge group and particle content, the proposed supersymmetric model has the following desirable features. (1) The conservation of baryon number (B) is 
automatic. (2) The Higgs sector is minimal, consisting of just one bidoublet $\Phi_{12}$ and two $\mathrm{SU}(2)_{\mathrm{R}} \times \mathrm{U}(1)$ doublets $\Phi_{3,4}$. (3) Realistic mass matrices for both quarks and leptons are obtained. (4) Flavor-changing neutral currents (FCNC) are naturally suppressed at the electroweak energy scale, except for $u$ quarks through scalar exchange. (5) The conservation of lepton number (L) is violated only through the singlet $N$ and the fact that both $L^{c}$ and $\Phi_{4}$ have identical transformations and the term $\Phi_{3} \Phi_{4}$ is unavoidable. The only significant effect of this at low energies is the appearance of small Majorana masses for the neutrinos. Rare decays violating lepton number are possible, but they are suppressed by inverse powers of some higher mass scale. For example, the rate for $\mu \rightarrow$ eee through $\mathrm{Z}$ exchange is proportional to $\mu_{34}^{-4}$.

At or below the electroweak energy scale of $10^{2} \mathrm{GeV}$, the proposed left-right supersymmetric model has the same particle content of the minimal supersymmetric standard model (MSSM). Their gauge interactions are of course identical but their Yukawa and scalar interactions do have important differences. For example, the physical neutral Higgs boson corresponding to the breaking of the standard $\mathrm{SU}(2) \times \mathrm{U}(1)$, i.e. $\operatorname{Re} \phi_{1}^{0} \cos \beta+\operatorname{Re} \phi_{2}^{0} \sin \beta$, where $\tan \beta \equiv v_{2} / v_{1}$, couples to $\bar{u} u$ with strength proportional to $v_{2} v_{1}^{-1} m_{d} \times$ a factor due to $u^{c}-x^{c}$ mixing in this model, instead of the well-known $m_{u}$ in the MSSM. The detailed phenomenology of this and other effects will be given elsewhere.

At the electroweak energy scale, only the two doublets contained in $\Phi_{12}$ are possibly relevant to the Higgs potential $V$ of this model. Let $\Phi_{1,2}=\left(\phi_{1,2}^{+}, \phi_{1,2}^{0}\right)$, then $V$ is of the well-known form

$$
\begin{aligned}
V & =\mu_{1}^{2} \Phi_{1}^{\dagger} \Phi_{1}+\mu_{2}^{2} \Phi_{2}^{\dagger} \Phi_{2}+\mu_{12}^{2}\left(\Phi_{1}^{\dagger} \Phi_{2}+\Phi_{2}^{\dagger} \Phi_{1}\right) \\
& +\frac{1}{2} \lambda_{1}\left(\Phi_{1}^{\dagger} \Phi_{1}\right)^{2}+\frac{1}{2} \lambda_{2}\left(\Phi_{2}^{\dagger} \Phi_{2}\right)^{2}+\lambda_{3}\left(\Phi_{1}^{\dagger} \Phi_{1}\right)\left(\Phi_{2}^{\dagger} \Phi_{2}\right)+\lambda_{4}\left(\Phi_{1}^{\dagger} \Phi_{2}\right)\left(\Phi_{2}^{\dagger} \Phi_{1}\right)
\end{aligned}
$$

Using the supersymmetric constraints of the $\mathrm{SU}(2) \times \mathrm{SU}(2) \times \mathrm{U}(1)$ gauge group and taking into account [5] the cubic interactions of $\Phi_{1,2}$ with $\operatorname{Re} \phi_{3}^{0}$ after its spontaneous breakdown to 
the standard $\mathrm{SU}(2) \times \mathrm{U}(1)$, the above quartic scalar couplings are determined to be

$$
\lambda_{1}=\lambda_{2}=\frac{1}{4}\left(g_{1}^{2}+g_{2}^{2}\right), \quad \lambda_{3}=-\frac{1}{4} g_{1}^{2}+\frac{3}{4} g_{2}^{2}, \quad \lambda_{4}=-g_{2}^{2},
$$

where $g_{1}$ and $g_{2}$ are the $\mathrm{U}(1)$ and $\mathrm{SU}(2)$ gauge couplings of the standard model respectively. Note that $\lambda_{3,4}$ are not the same as in the MSSM where they are equal to $\left(-g_{1}^{2}+g_{2}^{2}\right) / 4$ and $-g_{2}^{2} / 2$ respectively. We now have the tree-level sum rule

$$
m_{H^{ \pm}}^{2}=m_{A}^{2}+2 M_{W}^{2}
$$

where $m_{A}$ is the mass of the pseudoscalar boson, instead of the well-known $m_{H^{ \pm}}^{2}=m_{A}^{2}+M_{W}^{2}$ in the MSSM. The other tree-level sum rule

$$
m_{H_{1}^{0}}^{2}+m_{H_{2}^{0}}^{2}=m_{A}^{2}+M_{Z}^{2}
$$

remains the same, but radiative corrections due to the Yukawa couplings of the Higgs bosons will be different in this model. Note also that even though there are no cubic terms in the superpotential, $V$ does not reduce to that of the MSSM as in previous examples [6]. The reason is that in this model, the soft supersymmetry-breaking terms of the Higgs potential at the $\mathrm{SU}(2) \times \mathrm{SU}(2) \times \mathrm{U}(1)$ level must be chosen to maintain the hierarchy $v_{1,2} \ll v_{3}$ in such a way that the two scales cannot be entirely separated. As a result,

$$
m_{A}^{2}=-\frac{M_{W_{R}}^{2}}{\cos 2 \beta}
$$

and the Higgs sector reduces at the electroweak energy scale to that of a single physical particle as in the standard model. Hence $\cos 2 \beta<0$, or equivalently $\tan \beta>1$, is required in this model. Details will be given elsewhere.

Lepton number is violated here either because the singlet $N$ has a Majorana mass or because the terms $\Phi_{12} L L^{c}, \Phi_{12} \Phi_{4} L$, and $\Phi_{3} \Phi_{4}$ must coexist. Its effect is very much suppressed for ordinary processes. One important consequence for future experimental searches for 
supersymmetry is that there is no longer a "lightest supersymmetric particle." The photino for example will mix with the neutrinos and will decay. However, if the mixing turns out to be very small and the photino mass not too heavy, it may still be stable within the detector in a typical high-energy physics experiment.

Finally, it should be mentioned that if left-right exchange symmetry is desired, we can easily add two $\mathrm{SU}(2)_{\mathrm{L}}$ Higgs doublets $\Phi_{5,6} \sim(1,2,1, \pm 1 / 2)$ without affecting anything essential in this paper as long as $\Phi_{6}$, which transforms identically to $L$, does not have any vacuum expectation value. A conundrum of the MSSM is that $\Phi_{1}$ and $L$ transform identically and yet $\Phi_{1}$ must have a vacuum expectation value, but $L$ must not. Here the only superfields which need to have vacuum expectation values are $\Phi_{12}$ and $\Phi_{3}$, both of which are in representations different from the leptons.

\section{ACKNOWLEDGEMENT}

The author thanks V. Barger for discussions and for reading the manuscript. This work was supported in part by the U. S. Department of Energy under contracts No. DE-AT0387ER40327 and No. DE-AC02-76ER00881, by the Texas National Laboratory Research Commission, and by the University of Wisconsin Research Committee with funds granted by the Wisconsin Alumni Research Foundation. 


\section{References}

[1] See for example V. Barger, G. F. Giudice, and T. Han, Phys. Rev. D40, 2987 (1989); and references therein.

[2] E. Ma and P. Roy, Phys. Rev. D41, 988 (1990); E. Ma and D. Ng, ibid. D41, 1005 (1990).

[3] See for example S. Dimopoulos, R. Esmailzadeh, L. J. Hall, and G. D. Starkman, Phys. Rev. D41, 2099 (1990); and references therein.

[4] E. Ma, Phys. Rev. D36, 274 (1987); K. S. Babu, X.-G. He, and E. Ma, ibid. D36, 878 (1987).

[5] E. Ma and D. Ng, Univ. of Calif., Riverside Report No. UCRHEP-T103 (1993).

[6] E. Ma and D. Ng, Univ. of Calif., Riverside Report No. UCRHEP-T107 (1993); T. V. Duong and E. Ma, Univ. of Calif., Riverside Report No. UCRHEP-T111 (1993). 